\title{
Correction to: Relationship between tumor biomarkers and efficacy in MARIANNE, a phase III study of trastuzumab emtansine \pm pertuzumab versus trastuzumab plus taxane in HER2-positive advanced breast cancer
}

\author{
Edith A. Perez ${ }^{1 *}$ D, Sanne Lysbet de Haas ${ }^{2}$, Wolfgang Eiermann³ ${ }^{3}$ Carlos H. Barrios ${ }^{4}$, Masakazu Toi ${ }^{5}$,

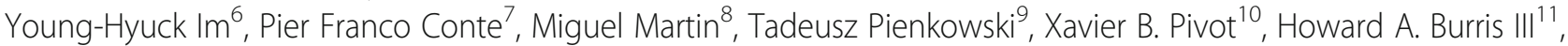
Sven Stanzel ${ }^{2}$, Monika Patre ${ }^{2}$ and Paul Anthony Ellis ${ }^{12}$

\section{Correction to: BMC Cancer https://doi.org/10.1186/s12885-019-5687-0}

Following publication of the original article [1], the authors reported the following errors in the article.

1) In Table 2, the layout has been updated. The corrected Table 2 is supplied below:

2) The legend for Fig. 3 has been adapted for clearer readability. The updated legend is as follows:

3) The competing interests statement has been updated below.

\section{Competing interests}

EAP was a salaried employee of Genentech, Inc. at the time this work was prepared and owns stock in F. Hoffmann-La Roche Ltd. SLdH, SS, and MP are salaried employees of F. Hoffmann-La Roche Ltd. SS and MP own stock in F. Hoffmann-La Roche Ltd. WE has served as a consultant and on Speakers' Bureaus for F. Hoffmann-La Roche Ltd. CHB has served as a consultant for F. Hoffmann-La Roche Ltd., Pfizer, GlaxoSmithKline, Novartis, Boehringer Ingelheim, and Eisai and has received research funding from Amgen, AstraZeneca, Boehringer Ingelheim, GlaxoSmithKline, Novartis, Pfizer, F. Hoffmann-La Roche/Genentech, Eisai, Lilly, Sanofi-Aventis, and Celgene. MT has received research funding from Chugai Pharmaceutical. PFC has served on Speakers' Bureaus for Novartis, F. Hoffmann-La Roche Ltd., and AstraZeneca and has received research funding from F. Hoffmann-La Roche Ltd. and Novartis. MM has received honoraria from and has served as a consultant for $F$. HoffmannLa Roche Ltd. TP has received honoraria and research funding from F. Hoffmann-La Roche Ltd., Pfizer, and Novartis. He has also served as a consultant for F. Hoffmann-La Roche Ltd. XBP has received honoraria from F. Hoffmann-La Roche Ltd., GlaxoSmithKline, Amgen, Novartis, Pierre Fabre, and
Eisai. He has also served as a consultant for F. Hoffmann-La Roche Ltd., Amgen, Novartis, Pierre Fabre, and Eisai. Y-HI, HAB, and PAE have nothing to disclose.

\section{Author details}

${ }^{1}$ Mayo Clinic, 4500 San Pablo Rd. S, Jacksonville, FL 32224, USA. ${ }^{2}$ F. Hoffmann-La Roche Ltd, Grenzacherstrasse 124, 4070 Basel, Switzerland. ${ }^{3}$ Interdisciplinary Oncology Center, Nussbaumstrasse 12, 80336 Munich, Germany. ${ }^{4}$ PUCRS School of Medicine, Av. Ipiranga 6681, Porto Alegre, RS 90619-900, Brazil. ${ }^{5}$ Graduate School of Medicine, Kyoto University, Yoshida-Konoe-cho, Sakyo-ku, Kyoto 606-8501, Japan. ${ }^{6}$ Samsung Medical Centre, 81 Irwon-Ro Gangnam-gu, Seoul 06351, South Korea. 'Department of Surgery, Oncology and Gastroenterology, University of Padova and Istituto Oncologico Veneto, Via Gattamelata 64, 35128 Padova, Italy. ${ }^{8}$ Instituto de Investigacion Sanitaria Gregorio Marañón, CIBERONC, GEICAM, Universidad Complutense, Avda. de Séneca, 2, 28040 Madrid, Spain. ${ }^{9}$ Postgraduate Medical Education Center, ul. Marymoncka 99, 02-813 Warsaw, Poland. ${ }^{10}$ Paul Strauss Cancer Center, 3 Rue de la Porte de l'Hôpital, BP 30042, 67065 Strasbourg, France. ${ }^{11}$ Sarah Cannon Research Institute and Tennessee Oncology, PLLC, 250 25th Ave N, Nashville, TN 37203, USA. ${ }^{12}$ Guys Hospital and Sarah Cannon Research Institute, Great Maze Pond, London SE1 9RT, UK.

Received: 13 June 2019 Accepted: 13 June 2019

Published online: 24 June 2019

\section{Reference}

1. Perez, et al. Relationship between tumor biomarkers and efficacy in MARIANNE, a phase III study of trastuzumab emtansine \pm pertuzumab versus trastuzumab plus taxane in HER2- positive advanced breast cancer. BMC Cancer. 2019:19:517 https://doi.org/10.1186/s12885-019-5687-0.

* Correspondence: Perez.edith@mayo.edu

${ }^{1}$ Mayo Clinic, 4500 San Pablo Rd. S, Jacksonville, FL 32224, USA

Full list of author information is available at the end of the article

(c) The Author(s). 2019 Open Access This article is distributed under the terms of the Creative Commons Attribution 4.0 International License (http//creativecommons.org/licenses/by/4.0/) which permits unrestricted use, distribution, and reproduction in any medium, provided you give appropriate credit to the original author(s) and the source, provide a link to the Creative Commons license, and indicate if changes were made. The Creative Commons Public Domain Dedication waiver (http://creativecommons.org/publicdomain/zero/1.0/) applies to the data made available in this article, unless otherwise stated. 
Table 2 Progression-free survival by HER2 expression subgroups

\begin{tabular}{|c|c|c|c|c|c|c|c|c|c|}
\hline & \multicolumn{2}{|c|}{$\begin{array}{l}\text { Trastuzumab + taxane } \\
\text { (Control) }\end{array}$} & \multicolumn{3}{|l|}{$\begin{array}{l}\text { T-DM1 } \\
\text { (T-DM1) }\end{array}$} & \multicolumn{4}{|c|}{$\begin{array}{l}\text { T-DM1 + pertuzumab } \\
(\mathrm{T}-\mathrm{DM} 1+\mathrm{P})\end{array}$} \\
\hline & $\begin{array}{l}\text { No. patients / No. } \\
\text { patients with PFS } \\
\text { event }\end{array}$ & $\begin{array}{l}\text { Median } \\
\text { PFS } \\
\text { (mo) }\end{array}$ & $\begin{array}{l}\text { No. patients / No. } \\
\text { patients with PFS } \\
\text { event }\end{array}$ & $\begin{array}{l}\text { Median } \\
\text { PFS } \\
\text { (mo) }\end{array}$ & $\begin{array}{l}\text { HR vs. } \\
\text { trastuzumab }+ \\
\text { taxane }(97.5 \% \mathrm{Cl})^{\mathrm{a}}\end{array}$ & $\begin{array}{l}\text { No. patients / No. } \\
\text { patients with PFS } \\
\text { event }\end{array}$ & $\begin{array}{l}\text { Median } \\
\text { PFS } \\
\text { (mo) }\end{array}$ & $\begin{array}{l}\text { HR vs. } \\
\text { trastuzumab + } \\
\text { taxane }(97.5 \% \text { Cl) }\end{array}$ & $\begin{array}{l}\text { HR vs. T-DM1 + } \\
\text { placebo }(97.5 \% \\
\text { Cl) }\end{array}$ \\
\hline \multicolumn{10}{|l|}{ All patients ${ }^{\mathrm{b}}$} \\
\hline IHC 3+ & $333 / 209$ & 14.4 & $340 / 215$ & 14.6 & $0.93(0.75-1.16)$ & $331 / 195$ & 16.7 & $0.83(0.67-1.04)$ & $0.90(0.72-1.12)$ \\
\hline IHC 2+ & $27 / 19$ & 12.6 & $25 / 20$ & 7.3 & $1.13(0.55-2.32)$ & $29 / 20$ & 8.3 & $1.25(0.61-2.59)$ & $0.98(0.48-2.02)$ \\
\hline \multicolumn{10}{|c|}{ IHC 2+/3+ patients combined ${ }^{C}$} \\
\hline $\begin{array}{l}\text { Focal IHC 2+/3+ } \\
(10-29 \%)^{d}\end{array}$ & $14 / 8$ & 12.4 & $12 / 10$ & 6.4 & $1.51(0.52-4.40)$ & $15 / 12$ & 7.5 & $1.41(0.50-3.94)$ & $1.00(0.38-2.65)$ \\
\hline $\begin{array}{l}\text { Heterogeneous } \\
\text { IHC } 2+/ 3+(30- \\
79 \%)\end{array}$ & $35 / 27$ & 10.6 & $37 / 25$ & 8.3 & $1.04(0.55-1.94)$ & $33 / 20$ & 6.3 & $1.11(0.57-2.17)$ & $0.91(0.46-1.78)$ \\
\hline $\begin{array}{l}\text { Homogeneous } \\
\text { IHC } 2+/ 3+ \\
(\geq 80 \%)\end{array}$ & $311 / 193$ & 14.6 & $316 / 200$ & 14.7 & $0.92(0.74-1.16)$ & $312 / 183$ & 17.8 & $0.82(0.65-1.04)$ & $0.89(0.71-1.13)$ \\
\hline \multicolumn{10}{|l|}{ IHC 3+ patients only } \\
\hline $\begin{array}{l}\text { Focal IHC 3+ } \\
(10-29 \%)^{d}\end{array}$ & $9 / 5$ & 8.3 & $11 / 7$ & 8.3 & $1.20(0.32-4.50)$ & $8 / 7$ & 4.2 & $5.11(0.99-26.40)$ & $2.28(0.60-8.71)$ \\
\hline $\begin{array}{l}\text { Heterogeneous } \\
\text { IHC 3+ (30- } \\
79 \%)\end{array}$ & $44 / 29$ & 10.5 & $45 / 34$ & 10.0 & $1.15(0.65-2.03)$ & 29/16 & 17.8 & $0.79(0.39-1.60)$ & $0.65(0.33-1.29)$ \\
\hline $\begin{array}{l}\text { Homogeneous } \\
\text { IHC 3+ ( } \geq 80 \%)\end{array}$ & $280 / 175$ & 14.6 & $284 / 174$ & 15.2 & $0.89(0.70-1.14)$ & $294 / 172$ & 17.7 & $0.82(0.65-1.05)$ & $0.92(0.73-1.17)$ \\
\hline
\end{tabular}

\section{anstratified hazard ratio}

${ }^{\mathrm{b}}$ Five patients with IHC $0 / 1+$ and five patients with unknown IHC status are not included in this table

${ }^{c}$ Categories were based on IHC subgroup and then combined

${ }^{\mathrm{d} C o m p a r e d}$ with the overall population, samples with focal HER2 expression were more likely to express mutated PIK3CA and lower levels of HER2 mRNA $\mathrm{Cl}$ confidence interval, HER2 human epidermal growth factor receptor 2, HR hazard ratio, IHC immunohistochemistry, NE not estimable, $P$ pertuzumab,

PFS progression-free survival, PIK3CA phosphoinositide 3-kinase catalytic subunit alpha, T-DM1 trastuzumab emtansine

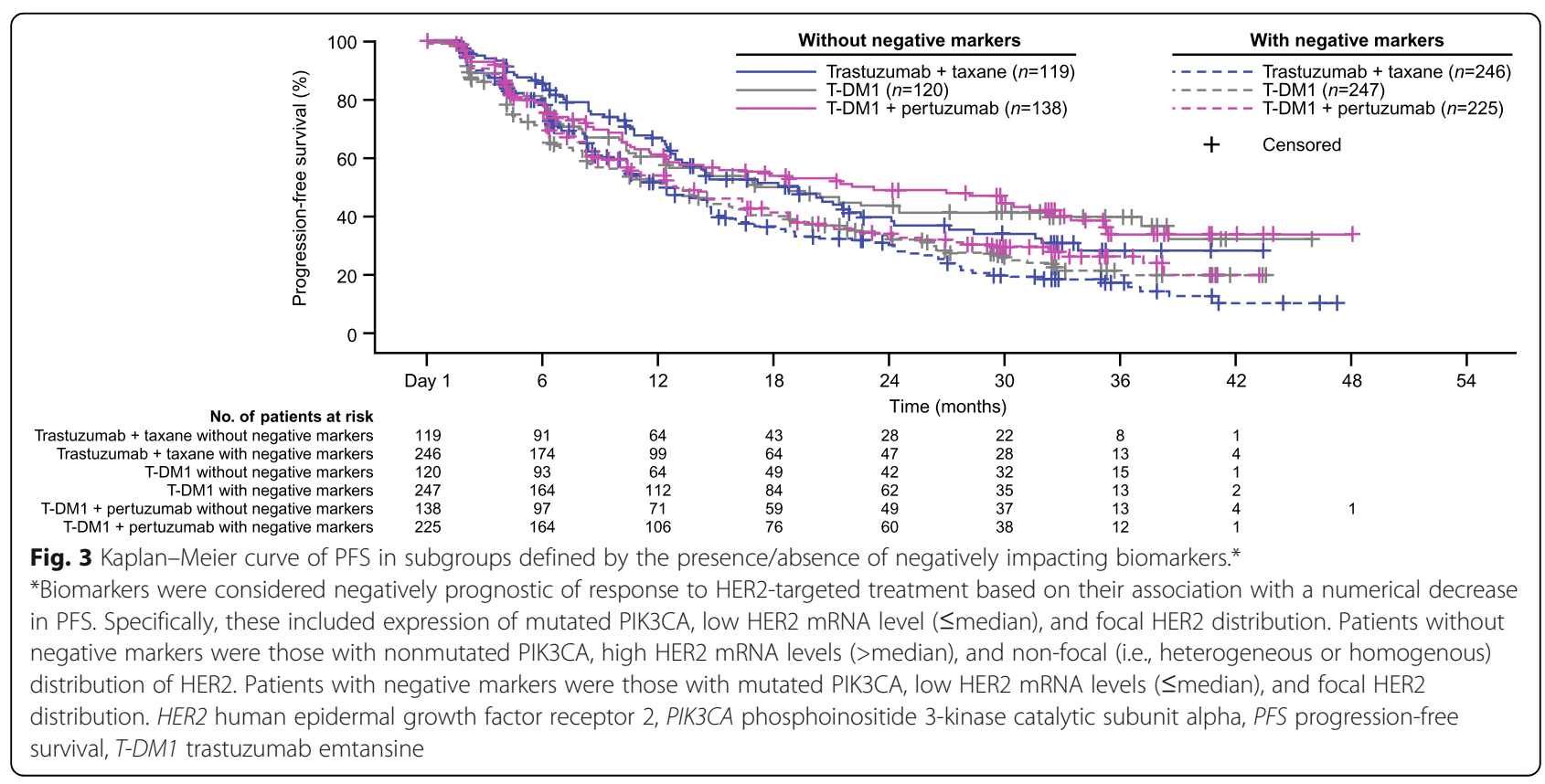

